\title{
Generalización de un método de integración a través de factorizaciones
}

\author{
Generalization of a method of integration through factoring \\ Jaime Castro Pérez, Andrés González Nucamendi, Gerardo Pioquinto Aguilar Sánchez \\ Departamento de Física y Matemáticas, Tecnológico de Monterrey, Ciudad de México, México \\ Correos-e: \{jcastrop, anucammen, gerardo.aguilar\}@itesm.mx
}

\begin{abstract}
Resumen- Como se sabe, cada método de integración sólo funciona para ciertos tipos o clases de funciones y no existe un método capaz de expresar la integral indefinida de cualquier función en términos de funciones elementales. En este artículo, mostramos una alternativa, basada en factorizaciones, para integrar cierta clase de funciones que, en ciertos casos, puede resultar una mejor herramienta que los métodos comúnmente usados. Como una aplicación de esa técnica resolvemos una clase particular de ecuaciones diferenciales del tipo $\frac{d P / d t}{P}=k f(P)$, donde $\frac{d P / d t}{P}$ es la tasa de crecimiento relativa de $P$ respecto a $t$. También resolvemos ejemplos para ilustrar este método y compararlo con los resultados que se obtienen utilizando Wolfram Alpha y una calculadora TI.
\end{abstract}

Abstract- As well known, each technique of integration is useful for a type or kind of functions and it doesn't exist a technique be able to express the indefinite integral of any function in terms of elementary functions. In this paper we show an alternative technique of integration based on factoring in order to obtain the indefinite integral for a class of functions, which sometimes, may be better tool than the conventional techniques of integration. As an application of this technique we solve a particular class of differential equations like $\frac{d P / d t}{P}=k f(P)$ where $\frac{d P / d t}{P}$ is the relative growth rate of $P$ with respect. Some examples are solved to illustrate this technique and their results are compared with the results obtained using Wolfram Alpha and TI calculator.

Key Word- Differential equation, Relative growth rate, Techniques of integration, Logistic Model

\section{INTRODUCCIÓN}

Cada método de integración logra integrar funciones de cierto tipo, pero no existe un método general para integrar cualquier función. Por el contrario, existen funciones que pueden ser integradas por diversos métodos.
Por ejemplo, en el modelo de crecimiento logístico de VerhulstPearl [5], que puede expresarse como

$$
\frac{d P / d t}{P}=k(M-P)
$$

Según el cual la tasa de crecimiento relativa $\frac{d P / d t}{P}$ respecto al tiempo $t$ de una población no es una constante como en el crecimiento exponencial, sino que es proporcional a la diferencia entre el tamaño actual $P$ de la población y un valor máximo denotado por $M$. Al separar variable para resolver la ecuación (1) se obtiene.

$$
\int \frac{d P}{P(M-P)}=\int k d t
$$

El lado izquierdo de esta ecuación diferencial se calcula tradicionalmente mediante el método de fracciones parciales [1], [2] y se obtiene

$$
\int \frac{d P}{P(M-P)}=-\frac{1}{M}(\ln (P-M)-\ln P)+C
$$

Sin embargo, también es posible seguir el siguiente método. Primero rescribimos la integral:

$\int \frac{d P}{P(M-P)}=\int \frac{d P}{P^{2}\left(M P^{-1}-1\right)}=\int \frac{P^{-2} d P}{M P^{-1}-1}$

Ahora sustituimos $u=M P^{-1}-1, d u=-M P^{-2} d P$ con lo que obtenemos

$$
\int \frac{d P}{P(M-P)}=-\frac{1}{M} \ln \left|M P^{-1}-1\right|+C
$$

La solución para la ecuación diferencial (1) puede expresarse como una función $P(t)$ que contiene tres parámetros $B, C$ y $M$ y que se le conoce como función logística. 


$$
P(t)=\frac{M}{1+C e^{-B t}}
$$

El modelo de crecimiento logístico es un caso particular de ecuaciones del tipo puede generalizarse como

$$
\frac{d P / d t}{P}=k f(P)
$$

Donde $f(P)$ representa una función de $P$ que en el modelo logístico corresponde a una función lineal $f(P)=b_{0}+b_{1} P$, pero que puede ser cualquier función con la que incluso se pueden modelar efectos estacionales. Esta ecuación diferencial, que se adopta en varios modelos demográficos de animales, se le conoce como hipótesis de dependencia de densidad [3]. Una clase particular de ecuaciones diferenciales es este tipo (3) nos lleva a las integrales que se resuelven mediante factorizaciones atípicas en la sección 2.1, mismas que resolvemos para una amplia gama de funciones $f(P)$. En las siguientes secciones éste método se generaliza a otro tipo de integrales.

\section{CONTENIDO}

A continuación, desarrollaremos tres casos e ilustramos con algunos ejemplos.

\subsection{Integrales de la forma $\int \frac{d u}{a u+b u^{\alpha}}$ donde $\alpha, \boldsymbol{a}, \boldsymbol{b} \in \mathcal{R}$ y $\boldsymbol{u}$ es una función de la variable $t$.}

Consideremos la siguiente ecuación diferencial

$$
\frac{d u}{d t}=k u\left(a+b u^{\alpha-1}\right)
$$

Si $\alpha=2$ y $b=1$ obtenemos el modelo logístico de VerhustPearl, por lo que podemos considerar la anterior ecuación como una generalización de ese modelo. La solución de ésta ecuación diferencial nos lleva de manera natural a integrales de la forma

$$
\int \frac{d u}{a u+b u^{\alpha}}
$$

Nótese que para $\alpha=1$, la integral es trivial, por lo tanto podemos suponer que $\alpha \neq 1$.

Para el caso general, reescribimos la integral de la siguiente forma

$$
\begin{gathered}
\int \frac{d u}{a u+b u^{\alpha}}=\int \frac{d u}{u^{\alpha}\left(\frac{a}{u^{\alpha-1}}+b\right)} \\
\int \frac{d u}{u^{\alpha}\left(a u^{1-\alpha}+b\right)}=\int \frac{u^{-\alpha}}{\left(a u^{1-\alpha}+b\right)} d u
\end{gathered}
$$

Esta última integral es inmediata ya que:

$$
\begin{gathered}
\int \frac{u^{-\alpha}}{\left(a u^{1-\alpha}+b\right)} d u=\frac{1}{a(1-\alpha)} \int \frac{a(1-\alpha) u^{-\alpha}}{\left(a u^{1-\alpha}+b\right)} d u \\
=\frac{1}{a(1-\alpha)} \ln \left|a u^{1-\alpha}+b\right|+C
\end{gathered}
$$

Así la ecuación diferencial generalizada (4) se reduce a

$$
\frac{1}{a(1-\alpha)} \ln \left|a u^{1-\alpha}+b\right|+C=k t
$$

Equivalentemente

$u(t)=\frac{1}{\left(B+D e^{-K t}\right)^{\frac{1}{\alpha-1}}}$

Donde $B=-\frac{b}{a}, \quad D=\frac{1}{a} e^{C a(1-\alpha)}, \quad K=a(\alpha-1) k$

Observe que si $\alpha=2$ y $B=1$ obtenemos el modelo logístico.

Ejemplo 1. Calcule

$$
\int \frac{d x}{(\sqrt{3}) x+(\sqrt{2}) x^{\pi}}
$$

Es claro que este ejemplo no es por fracciones parciales ni por ninguno de los métodos tradicionales de integración.

Aplicamos el nuestro método alternativo y con $\alpha=\pi, a=\sqrt{3}$ $b=\sqrt{2}$ y obtenemos que

$$
\begin{gathered}
\int \frac{d x}{(\sqrt{3}) x+(\sqrt{2}) x^{\pi}}= \\
\frac{1}{\sqrt{3}(1-\pi)} \ln \left|\sqrt{3} x^{1-\pi}+\sqrt{2}\right|+C
\end{gathered}
$$

Compare este resultado con el obtenido mediante Maple V:

$$
\int \frac{d x}{(\sqrt{3}) x+(\sqrt{2}) x^{\pi}}=
$$

$$
\frac{\sqrt{3}}{6(\pi-1)}\left(\ln 2-2 \ln \left(\sqrt{3} x+\sqrt{2} x^{\pi}\right)+2 \pi \ln x\right)+C
$$

El resultado obtenido mediante wolframalpha.com es similar al nuestro:

$$
\int \frac{1}{\sqrt{3} x+\sqrt{2} x^{\pi}} d x=\frac{\pi \log (x)-\log \left(\sqrt{2} x^{\pi}+\sqrt{3} x\right)}{\sqrt{3}(\pi-1)}
$$

Ejemplo 2. En este ejemplo tenemos que $\alpha=\sqrt{2}, a=3$ 
$b=4$ у $u=e^{x}$

$$
\begin{gathered}
\int \frac{e^{x} d x}{3 e^{x}+4 e^{x \sqrt{2}}} \\
=\frac{1}{3(1-\sqrt{2})} \ln \left(3 e^{x-x \sqrt{2}}+4\right)+C
\end{gathered}
$$

Con maple V obtenemos:

$$
-\frac{1}{3(\sqrt{2}-1)}\left(\ln \left(e^{x \sqrt{2}}+\frac{3}{4} e^{x}\right)-x \sqrt{2}\right)+C
$$

En wolframalpha.com, obtenemos:

$$
\frac{1}{3}(1+\sqrt{2})\left(x \sqrt{2}-\ln \left((1+\sqrt{2})\left(3 e^{x}+4 e^{x \sqrt{2}}\right)\right)\right)+C
$$

Pero esta vez, este paquete no da la opción a ver la solución paso a paso.

Nótese también que es posible resolver esta integral de la siguiente forma:

$$
\begin{gathered}
\int \frac{e^{x}}{3 e^{x}+4 e^{x \sqrt{2}}} d x=\int \frac{e^{x}}{3 e^{x}+4 e^{x \sqrt{2}}}\left(\frac{e^{-x \sqrt{2}}}{e^{-x \sqrt{2}}}\right) d x \\
=\int \frac{e^{x-x \sqrt{2}}}{3 e^{x-x \sqrt{2}}+4} d x=\frac{1}{3(1-\sqrt{2})} \ln \left(3 e^{x-x \sqrt{2}}+4\right)+C
\end{gathered}
$$

Es el mismo resultado obtenido con nuestro método alternativo.

\subsection{Integrales de la forma $\int \frac{d u}{\sqrt{a u^{\alpha+1}+b u^{2 \alpha}}}$ donde $\alpha, a, b \in \mathcal{R}$} y $u$ es una función de la variable " $x$ "

Nuevamente si $\alpha=1$, entonces la integral es trivial. Por lo tanto supongamos que $\alpha \neq 1$. Así podemos escribir la integral anterior de la siguiente forma:

$$
\begin{aligned}
\int \frac{d u}{\sqrt{a u^{\alpha+1}+b u^{2 \alpha}}}=\int \frac{d u}{\sqrt{u^{2 \alpha}\left(a u^{1-\alpha}+b\right)}} \\
=\int \frac{d u}{u^{\alpha} \sqrt{a u^{1-\alpha}+b}} \\
=\int \frac{u^{-\alpha} d u}{\sqrt{a u^{1-\alpha}+b}}
\end{aligned}
$$

Notamos que esta integral es inmediata ya que:

$$
\begin{aligned}
& \int \frac{u^{-\alpha} d u}{\sqrt{a u^{1-\alpha}+b}} \\
= & \frac{1}{a(1-\alpha)} \int \frac{a(1-\alpha) u^{-\alpha} d u}{\sqrt{a u^{1-\alpha}+b}} \\
= & \frac{2}{a(1-\alpha)} \sqrt{a u^{1-\alpha}+b}+C
\end{aligned}
$$

Ejemplo 3. Calcule

$$
\int \frac{d x}{\sqrt{5 x^{\sqrt{2}+1}+3 x^{2 \sqrt{2}}}}
$$

Aplicamos el método alternativo con $\alpha=\sqrt{2}, a=5, b=3$ y obtenemos:

$$
\begin{gathered}
\int \frac{d x}{\sqrt{5 x^{\sqrt{2}+1}+3 x^{2 \sqrt{2}}}} \\
=\frac{2}{5(1-\sqrt{2})} \sqrt{5 x^{1-\sqrt{2}}+3}+C
\end{gathered}
$$

En Maple V, obtenemos

$$
\frac{6}{\sqrt{5 x^{1+\sqrt{2}}-3 x^{2 \sqrt{2}}}} \frac{x^{\sqrt{2}}-\frac{5}{3} x}{5 \sqrt{2}-5}+C
$$

Mientras que con wolframalpha, obtenemos (sin opción paso a paso)

$$
=-\frac{2}{5}(1+\sqrt{2}) x^{-\sqrt{2}} \sqrt{x^{\sqrt{2}}\left(5 x-3 x^{\sqrt{2}}\right)}+C
$$

\subsection{Integrales de la forma $\int \frac{d u}{\sqrt[n]{a u^{\alpha(n-1)+1}+b u^{n \alpha}}}$ donde \\ $\alpha, a, b \in \mathcal{R}$ y $u$ es una función de la variable " $x$ "}

Igual que antes, si $\alpha=1$, entonces la integral es trivial. Por lo tanto supongamos que $\alpha \neq 1$. Asi podemos escribir la integral anterior de la siguiente forma:

$$
\begin{aligned}
& \int \frac{d u}{\sqrt[n]{a u^{\alpha(n-1)+1}+b u^{n \alpha}}} \\
& =\int \frac{d u}{\sqrt[n]{a u^{\alpha n-\alpha+1}+b u^{n \alpha}}} \\
& =\int \frac{d u}{\sqrt[n]{u^{n \alpha}\left(a u^{-\alpha+1}+b\right)}}
\end{aligned}
$$




$$
=\int \frac{d u}{u^{\alpha} \sqrt[n]{a u^{-\alpha+1}+b}}
$$

Notamos que esta última integral es inmediata ya que:

$$
\begin{gathered}
\int \frac{d u}{u^{\alpha} \sqrt[n]{a u^{-\alpha+1}+b}} \\
=\frac{1}{a(-\alpha+1)} \int \frac{a(-\alpha+1) u^{-\alpha} d u}{\sqrt[n]{a u^{-\alpha+1}+b}} \\
=\frac{1}{a(-\alpha+1)\left(-\frac{1}{n}+1\right)}\left[a u^{-\alpha+1}+b\right]^{-\frac{1}{n}+1}+C
\end{gathered}
$$

Ejemplo 4. Sean $\alpha=\sqrt{3}, a=2, b=7, n=3 \mathrm{y}$ consideramos la siguiente integal:

$$
\begin{gathered}
\int \frac{d x}{\sqrt[3]{2 x^{(2 \sqrt{3}+1)}+7 x^{3 \sqrt{3}}}} \\
=\int \frac{d x}{x^{\sqrt{3} \sqrt[3]{2 x^{-\sqrt{3}+1}+7}}} \\
=\frac{1}{2(-\sqrt{3}+1)\left(-\frac{1}{3}+1\right)}\left[2 x^{-\sqrt{3}+1}+7\right]^{-\frac{1}{3}+1}+C \\
=\frac{3}{4(-\sqrt{3}+1)}\left[2 x^{-\sqrt{3}+1}+7\right]^{\frac{2}{3}}+C
\end{gathered}
$$

En Maple V obtenemos:

$$
-21 \frac{\frac{2}{7} x+x^{\sqrt{3}}}{\sqrt[3]{2 x x^{2 \sqrt{3}}+7 x^{3 \sqrt{3}}(4 \sqrt{3}-4)}}+C
$$

En wolframalpha obtenemos (sin opción paso a paso):

$$
\frac{3 x^{-2 \sqrt{3}}\left[x^{2 \sqrt{3}}\left(7 x^{\sqrt{3}}+2 x\right)\right]^{\frac{2}{3}}}{4 \sqrt{3}-4}+C
$$

Para terminar con este trabajo consideremos la función continua en todos los números reales $F(x)=\int_{0}^{x} \frac{d t}{3 e^{t}+4 e^{\sqrt{2}} t} \quad \mathrm{y}$ planteamos

Ejemplo 5 Calcule

$$
\int e^{x} F(x) d x
$$

Para resolver esta integral utilizamos la integración por partes haciendo $u=F(x), d v=e^{x} d x$. Por lo tanto $d u=\frac{d x}{3 e^{x}+4 e^{\sqrt{2} x}}$ y $v=e^{x}$. Así tenemos

$$
\begin{gathered}
\int e^{x} F(x) d x \\
=e^{x} F(x)-\int \frac{e^{x} d x}{3 e^{x}+4 e^{\sqrt{2} x}}
\end{gathered}
$$

Ahora usamos el resultado del ejemplo 2 y obtenemos,

$$
=e^{x} F(x)+\frac{\ln \left(3 e^{x-\sqrt{2} x}+4\right)}{3(\sqrt{2}-1)}+C
$$

\section{DISCUSIÓN}

Al resolver las ecuaciones diferenciales que resultan del modelo logístico de Verhulst llegamos de manera natural a un cierto tipo de integrales que hemos resuelto usando nuestro método de integración.

Las funciones logísticas tienen una gran variedad de aplicaciones tanto en problemas de crecimiento demográfico [3] como en modelos de regresión no lineales conocidos como modelos Logit [2]. En ambos casos la solución (5) que hemos obtenido permite mejorar el ajuste a los datos observados si se le compara con la función logística que, como se hizo notar, resulta un caso particular de las funciones obtenidas.

\section{CONCLUSIONES}

Hemos presentado un método de integración basado en factorizaciones que, en primer lugar, puede evitar procedimientos de fracciones parciales demasiado largos, pero que además resuelven integrales que no corresponden típicamente a fracciones parciales. Además presentamos una generalización al modelo logístico de Verhulst como una aplicación. Comparamos las soluciones obtenidas con nuestro método con aquellas que se obtienen al integrar con Maple V, wolframalpfa.com y con TI-npire CAS. Remarcamos que con la TI no se pueden calcular ninguna de las integrales de nuestros ejemplos. 


\section{REFERENCIAS}

[1]. Alejandro Martínez A. (2006, Ago.). Descomposición en Fracciones Parciales. Scientia Et Technica, vol. XII, núm. 31, pp. 259-264. Universidad Tecnológica de Pereyra, Pereira, Colombia.

[2]. Damodar N. Gujarati, Dawn C. Porter (2010). Econometría. (5 ${ }^{\mathrm{a}}$ ed.). México: McGraw-Hill.2010, p. 553.

[3]. Dennis G. Zill. Ecuaciones Diferenciales con Aplicaciones de Modelado. (6 ${ }^{\mathrm{a}}$. Ed.).México: Thomson. 1997, p. 86.

[4]. Edwin J. Purcell, Dale Varberg, Steven E. Rigdom. Cálculo. México: Pearson Prentice-Hall. 2007, p. 404410.

[5]. Pearl R., Reed L. J. (1920, June 15). On the Rate of Growth of the Population, of United States since 1790 and its Mathematical Representation. Proceedings of the National Academy of Sciences. USA 6; 275-288. 